\title{
Wohl und Weh
}

\author{
Eine Ausstellung zur Geschichte des Basler Kinderspitals beleuchtet die Entwicklung \\ der Kindermedizin und Kinderkrankenpflege.
}

Sabine Braunschweig

Historikerin, Projektleiterin

Korrespondenz:

Sabine Braunschweig Büro für Sozialgeschichte Dornacherstrasse 192 CH-4053 Basel Tel. 0613311800

braunschweig@ sozialgeschichte-bs.ch
Im Jahr 1862 wurde in Basel das Kinderspital am Rhein als «Musteranstalt» eröffnet. Nun ist es Ende Januar in einen mustergültigen Neubau umgezogen. Bevor das alte Gebäude neuen Wohnblöcken weichen muss, öffnet es nochmals seine Tore und zeigt die Ausstellung «Wohl und Weh. Vom Kinderspitäli zum UKBB (Universitäts-Kinderspital beider Basel)». Auf vielfältige Weise wird die fast 150-jährige Geschichte dieses für die Region Nordwestschweiz bedeutsamen Areals illustriert.

Viele Jahrzehnte war das auf Privatinitiative gegründete Kinderspital ein familiär geführter Betrieb, der von Hauseltern verwaltet und von einem Arzt medizinisch geleitet wurde. Zunächst waren Diakonissen aus Riehen in der Pflege tätig, die dann all-

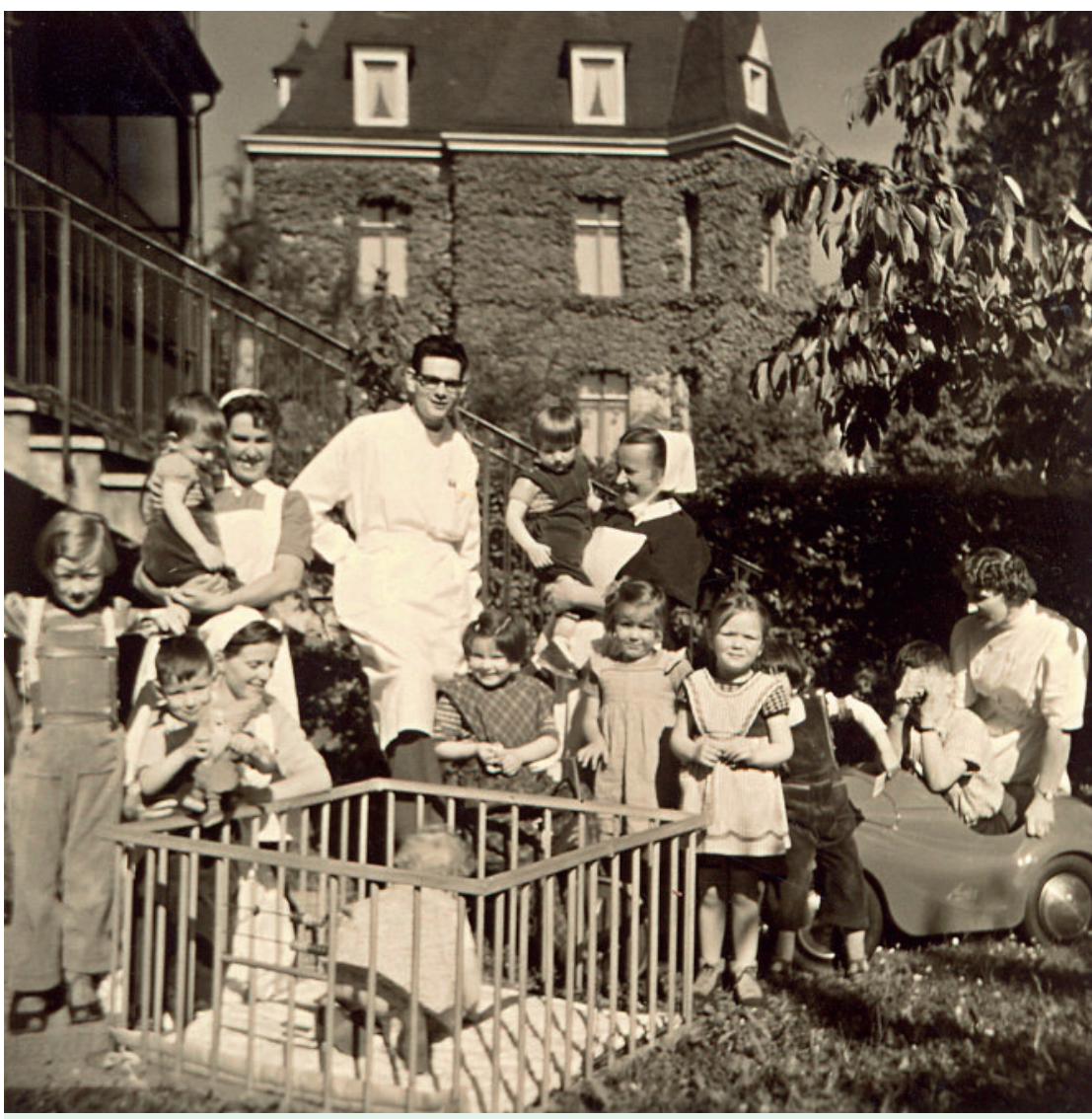

Während der Polioepidemie 1954 in Basel waren zahlreiche Kinder hospitalisiert (Privat).

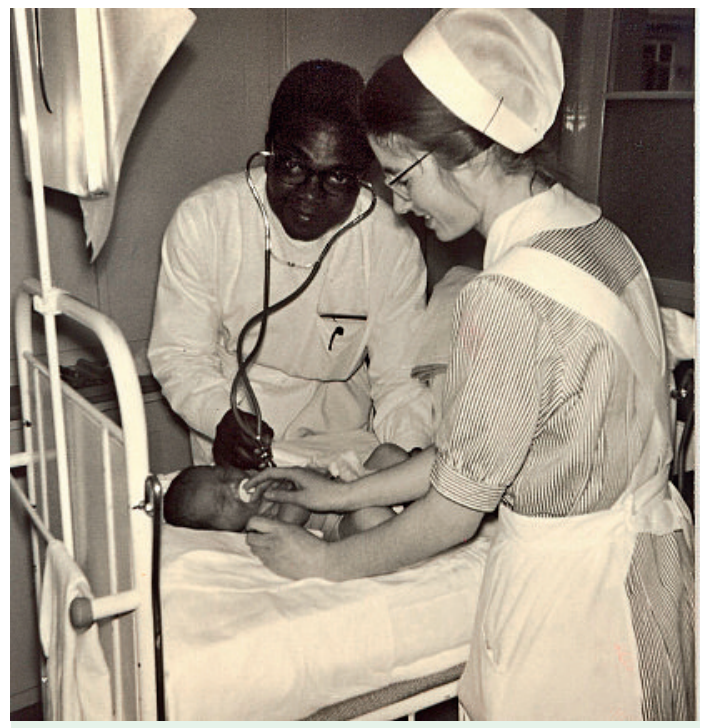

Seit den 1940er Jahren arbeiteten Ärztinnen und Ärzte aus dem Ausland am Kinderspital in Basel und trugen zu einem regen wissenschaftlichen Austausch bei. Hier ein Arzt aus dem Kongo 1963 (Fotoabteilung UKBB).

mählich von freien Kinderkrankenschwestern abgelöst wurden. Mit den Veränderungen in der Medizin, Chirurgie und Orthopädie sowie neuen pflegerischen und pädagogischen Erkenntnissen in der zweiten Hälfte des 20. Jahrhunderts entwickelte sich das Kinderspital zu einer professionell geführten staatlichen Institution. Immer wieder waren Kinderärzte und -ärztinnen aus Basel an wichtigen Forschungen beteiligt. So erwies sich für Basel 1939 die Wahl von Ernst Freudenberg (1884-1967), Professor für Kinderheilkunde an der Universität Marburg an der Lahn, der wegen der jüdischen Abstammung seiner Frau zwangsweise in den Ruhestand versetzt worden war, als grosse Chance. Seine Forschungen über Rachitis und Tetanie, über die Ernährung des Kindes oder über Stoffwechselkrankheiten verliehen ihm einen internationalen Ruf und brachten ihm zahlreiche Ehrungen ein. Er leitete die Redaktion des «Jahrbuchs für Kinderheilkunde», der ältesten kinderärztlichen Zeitschrift, die er zu den international anerkannten «Annales Paediatrici» umgestaltete.

Eine Pionierrolle nahm das Basler Kinderspital bei der Öffnung der Besuchszeiten ein. 1968 wurden sie von zweimal wöchentlich einer Stunde auf täglich 


\section{«Wohl und Weh»}

Die Ausstellung findet vom 17. Februar bis 17. April 2011 in der Poliklinik des alten Kinderspitals an der Römergasse 8 in Basel statt. Öffnungszeiten: Montag bis Freitag, 13.00 Uhr-17.00 Uhr, Samstag und Sonntag, 10.00-17.00 Uhr. Freier Eintritt.

Öffentliche Führungen werden jeden Sonntag um 11.00 Uhr angeboten. Private Führungen und Stadtrundgänge vom alten zum neuen Kinderspital können auch ausserhalb der Öffnungszeiten gebucht werden.

Das Begleitprogramm bietet pädiatrische, kinderpharmakologische und pflegerische Fachkolloquien an. Sie finden alle in der Aula des neuen UKBB, Spitalstrasse 33, statt:

\section{3. $2011,16.00 \mathrm{Uhr}-18.00 \mathrm{Uhr}$}

\section{Die Betreuung von Kindern und Jugendlichen im Spital}

1. Kolloquium des Fachbereichs Pflegeentwicklung

Was für ein Spital brauchen Kinder? - Die Entwicklung der Kinderrechte im Gesundheitswesen

Netty Fabian, Pflegeexpertin BScN, UKBB

Kinder gestalten ihr Spital: Partizipative Projekte im UKBB

Zeno Steuri, Geschäftsleiter Kinderbüro Basel

Kinderhospiz - ein Baustein in der Versorgungskette kranker Kinder in der Schweiz

Sabine Kraft, Geschäftsführerin Stiftung Kinderhospiz Basel

Patienten- und Familienedukation: Modischer Trend oder Notwendigkeit?

Ulrike Emmenegger, Pflegeexpertin BScN, UKBB

\section{3.2011 18.30 Uhr-20.00 Uhr}

\section{Kinder - Medikamente - Gift: Neues und Altes zum Themo}

Kolloquium zur Kinderpharmakologie

Medikamente und Kinder: eine Geschichte mit zwei Gesichtern

Markus Lampert, Dr. phil. II, Spitalapotheker Bruderholz

Vergiftungsfälle in der Geschichte der Kinderpharmakologie

Michael Kessler, Dr. phil. II, Leiter des Pharmazie-Historischen Museums

der Universität Basel

\section{3. 2011, 18.30-20.00 Uhr}

\section{Medizinische Entwicklungen - früher und heute}

Kolloquium des Fachbereichs Pädiatrie

Wohl und Weh in der Geschichte der Kindermedizin

Iris Ritzmann, PD Dr. med., lic. phil., wissenschaftliche Mitarbeiterin

des Medizinhistorischen Instituts der Universität Zürich

Infektionskrankheiten 2011 - von banal bis fatal

Ulrich Heininger, Prof. Dr. med., leitender Arzt des UKBB

Hasenscharte - Wolfsrachen? Die moderne ganzheitliche Behandlung angeborener Spaltbildungen des Gesichts

Katja Schwenzer-Zimmerer, PD Dr. med., Dr. dent., leitende Ärztin des Universitätsspitals Basel

Magenspiegelungen im aktuellen Spiegelbild

Raoul I. Furlano, Dr. med., Leiter der Pädiatrischen Gastroenterologie des UKBB

\subsubsection{1, 16.00-18.00}

\section{Kinderkrankenpflege - ein Berufsbild im Wandel}

2. Kolloquium des Fachbereichs Pflegeentwicklung

Vom Mutterersatz zur professionellen Kinderkrankenpflege

Sabine Braunschweig, Historikerin, Büro für Sozialgeschichte

Netzwerkkinder - ein Generationenvergleich

Miriam Engelhardt, Dr. phil., USB

Gesundheits- und berufspolitische Perspektiven

Isabel Kym, dipl. Pflegefachfrau - Intensivpflege, UKBB, Präsidentin der IG KJFF des SBK

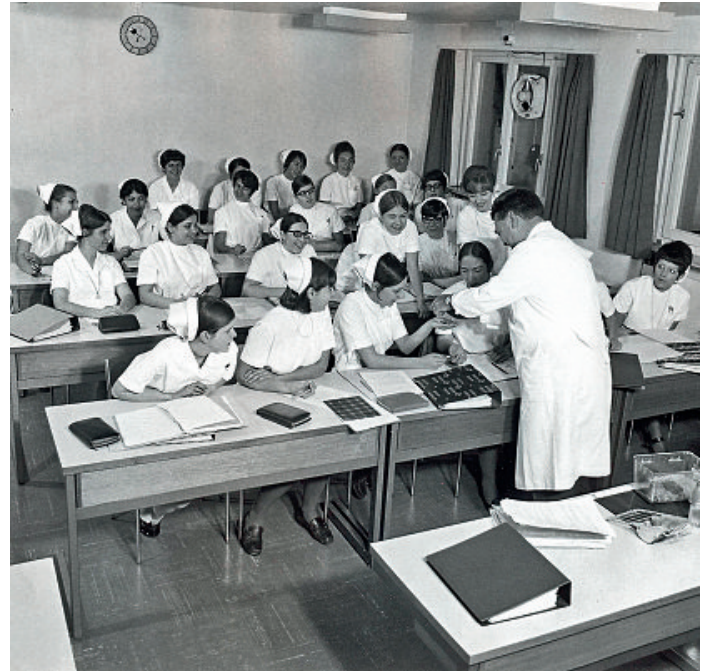

Ärzte unterrichteten die Schwesternschülerinnen an der eigenen Schule für Kinderkrankenpflege. Hier ein Arzt im Unterricht um 1965 (Fotograf: Peter Hemann).

vier Stunden ausgeweitet, und 1975 wurde die 24-stündige Besuchszeit eingeführt. Möglich machten diese Änderungen einerseits Erkenntnisse über das geringere Infektionsrisiko als angenommen, andererseits das Wissen um psychologische Bindungstheorien, die Anfang 1960er Jahre bekannt wurden. Auch

\section{Eine Pionierrolle nahm das Basler Kinderspital bei der Öffnung der Besuchszeiten ein.}

die Rolle der Kinderkrankenschwestern hatte sich verändert. So wurde nun die Behandlung und Pflege der kranken Kinder eine gemeinsame Aufgabe und ein gemeinsames Ziel des ärztlichen, pflegerischen und therapeutischen Personals sowie der Familienangehörigen.

Diese und zahlreiche weitere Themen greift die Ausstellung «Wohl und Weh» auf. Grundlage sind historische und aktuelle Objekte und Fotos aus der bedeutenden Sammlung von Vreni Kuhfuss, pensionierter Kinderkrankenschwester und Berufsschullehrerin. Bereichert wird die Ausstellung durch Hörstationen mit ehemaligen Patienten und Familienangehörigen, Pflegepersonen, Ärzten und Forscherinnen sowie historischen Filmsequenzen. Ein Begleitprogramm während der Ausstellung umfasst Führungen und Stadtrundgänge, Fachkolloquien und Aktivitäten für Kinder.

Weitere Informationen beim Verein Geschichte Kinderspital Basel,

Dornacherstrasse 192, 4053 Basel, Tel. 06133118 00,

wohlundweh@sozialgeschichte-bs.ch,www.sozialgeschichte-bs.ch 